Cite this: J. Mater. Chem. B, 2014, 2 , 2606

Received 11th December 2013 Accepted 28th January 2014

DOI: $10.1039 / c 3 t b 21763 a$

www.rsc.org/MaterialsB

\section{Selective functionalization of patterned glass surfaces}

\author{
E. Ploetz, ${ }^{a}$ B. Visser, ${ }^{b}$ W. Slingenbergh, ${ }^{\text {b }}$ K. Evers, ${ }^{b}$ D. Martinez-Martinez, ${ }^{b}$ Y. T. Pei, ${ }^{\text {b }}$ \\ B. L. Feringa, ${ }^{c}$ J. Th. M. De Hosson, ${ }^{b}$ T. Cordes ${ }^{\star a}$ and W. F. van Dorp ${ }^{\star b}$
}

Tailored writing and specific positioning of molecules on nanostructures is a key step for creating functional materials and nano-optical devices, or interfaces for synthetic machines in various applications. We present a novel approach for the selective functionalization of patterned glass surfaces with functional probes of any nature. The presented strategy is optimized for imaging fluorophore labeled nanostructures for (single-molecule) fluorescence microscopy. The first step in the protocol is coating a glass surface, here a microscope cover slide, with a $60 \mathrm{~nm}$ thick diamond-like carbon film. Subsequently, the pattern is defined by either writing silicon oxide on the coating with a focused electron beam, or by etching the coating with a focused ion beam to expose the glass surface. Finally, the pattern is silanized and functionalized. We demonstrate the selective binding of organic fluorophores and imaging with high contrast, especially in total-internal-reflection mode. The presented approach is flexible and combines bottom-up assembly with high-resolution lithography on glass cover slides to precisely position and image functional molecules of any type.

\section{Introduction}

Smart materials can change their properties under the influence of external stimuli and can potentially be used in medical, civil and military applications. ${ }^{1}$ Nature is a source of inspiration for developing such smart materials, since biological systems usually contain numerous complex functional components. For instance, myosin molecules, a broad family of motor proteins, generate force for muscle contraction ${ }^{2}$ and guanosine nucleotide-binding proteins transmit signals across cell membranes, acting as molecular switches..$^{3-5}$ The ability to mimic this allows us to produce advanced materials with responsive surfaces.

Recently, an increasing number of such functional molecules has been synthesized in laboratories, for instance, artificial molecular motors ${ }^{6-8}$ or electrical switches. ${ }^{9-11}$ Such molecules have been used for the light induced changing of the hydrophobicity, ${ }^{12,13}$ the conductance ${ }^{14}$ for inducing macroscopic mechanical deformation ${ }^{15}$ or for attaching cells or proteins on surfaces for therapeutic applications. ${ }^{1}$

However, if we want to exploit these molecules and functionalities for complex devices, we will have to interface the molecules with a surface and/or other components. ${ }^{16}$ One important issue is

${ }^{a}$ Molecular Microscopy Research Group \& Single-molecule Biophysics, Zernike Institute for Advanced Materials, University of Groningen, Nijenborgh 4, 9747 AG Groningen, The Netherlands. E-mail: t.m.cordes@rug.nl

${ }^{b}$ Applied Physics, Zernike Institute for Advanced Materials, University of Groningen, Nijenborgh 4, 9747 AG Groningen, The Netherlands. E-mail: w.f.van.dorp@rug.nl

${ }^{c}$ Stratingh Institute for Chemistry, Zernike Institute for Advanced Materials, University of Groningen, Nijenborgh 4, 9747 AG Groningen, The Netherlands to position them with high precision. For instance, the precise positioning of functional molecules is necessary in nanophotonics to couple optically active molecules with structures like nanoantennas ${ }^{17}$ or mechanical resonators. ${ }^{18}$ But while top-down lithography has progressed from the micrometer scale down to the nanometer scale and even below, ${ }^{19-21}$ several other issues remain to be solved. Do the molecules retain their functionality at that position on the surface? How do we verify both position and functionality at the molecular level? What are the practical requirements for constructing such systems?

Several strategies are available for selective deposition of functional molecules at the submicrometer scale. Most of them rely on self-assembled monolayers (SAMs) that cover a sample surface, comparable to a carpet. The SAM is then locally modified using electron beam lithography ${ }^{22}$ or scanning probe microscopy. ${ }^{23}$ The created pattern can be decorated with functional groups, while the remaining SAM carpet prevents undesired and unselective binding on the rest of the surface. Other strategies, such as dip-pen lithography ${ }^{24,25}$ and single-molecule cut-and-paste ${ }^{26}$ enable the selective decoration of surfaces with functional groups without any pre-treatment of the surface. We have recently presented a purely additive functionalization technique, which we called molecular assembly controlled by electron beam induced deposition (MACE-ID). ${ }^{27}$ MACE-ID allows us to precisely deposit siliconoxide $\left(\mathrm{SiO}_{x}\right)$ structures of defined size and shape on a substrate of choice, which can be selectively decorated with functional molecules. We demonstrated that it is possible to decorate structures as small as $25 \mathrm{~nm}$ selectively with fluorophores. ${ }^{27}$ 
However, our studies have shown that it is desirable for fluorescence applications to significantly increase the signal-tonoise ratio during fluorescence imaging of MACE-ID structures. In our previous work the $\mathrm{SiO}_{x}$ features were written on Si wafers and decorated with the fluorophore ATTO655. Since Si wafers are not optically transparent, they were imaged inverted on a microscope cover slide using a "sandwich" approach; the Si wafer was placed on a cover slide with the patterned surface facing down, being immersed in a buffer solution. This approach has several limitations. It is difficult to handle the sample and to change the buffer composition (necessary for changing the photophysical properties of fluorophores ${ }^{28,32,33}$ ) in the thin liquid film between the wafer and the cover slide. Most importantly, however, imaging is only possible in the epi-fluorescence mode. Here the signal-to-signal-to-background and -noise values are value is much lower than that in total internal reflection imaging mode (TIRF), used for e.g., single-molecule ${ }^{28}$ and localization-based super-resolution microscopy. ${ }^{29-33}$

To overcome these limitations, we here extend MACE-ID and present an alternative strategy that allows selective functionalization of nanopatterned microscope glass slides. The general strategy is shown in Fig. 1. A glass cover slide is coated with a thin layer of diamond-like carbon (DLC), see Fig. 1a. A pattern is defined on the coated glass by either locally depositing $\mathrm{SiO}_{x}$ with an electron beam (MACE-ID) or locally removing the DLC with an ion beam (Fig. 1b). In subsequent steps, the sample is silanized (Fig. 1c) and functionalized (Fig. 1d) with e.g., fluorophores. We demonstrate that the imaging of selectively labeled patterns with total internal reflection fluorescence (TIRF) microscopy is possible with these two strategies. Our new approach is flexible in the choice of (fluorescent) labels and gives precise control over the position of the molecular species.

\section{Materials and methods}

Unless stated otherwise, all chemical reagents were purchased from Sigma Aldrich and used as received without further purification. Standard microscope cover slides (Marienfeld, no. $1.5 \mathrm{H}$, high precision) are used as substrates for imaging and functionalization.

\subsection{Sample preparation}

DLC coating procedure. The glass cover slides are cleaned before coating. The slides are cleaned by sonication in a series of liquids $(1 \%$ Helmanex III solution, acetone, ethanol and finally $1 \mathrm{M} \mathrm{KOH}$ ), rinsing with milliQ water after each step. ${ }^{34}$ Subsequently, the slides are dried and cleaned in a plasma cleaner (Plasma Etch, PE-25-JW) for $10 \mathrm{~min}$. When stored the cover slides are kept under vacuum in a desiccator.

The DLC film is deposited with a Teer UDP400/4 closed field unbalanced magnetron sputtering system with all four magnetrons powered off. A pulsed DC power unit (Pinnacle plus, Advanced Energy) is used for substrate biasing, operating at $250 \mathrm{kHz}$ and $87.5 \%$ duty cycle. The base pressure of the vacuum chamber before the coating process is $2 \times 10^{-6} \mathrm{mbar}$.

\section{(a) DLC coating}

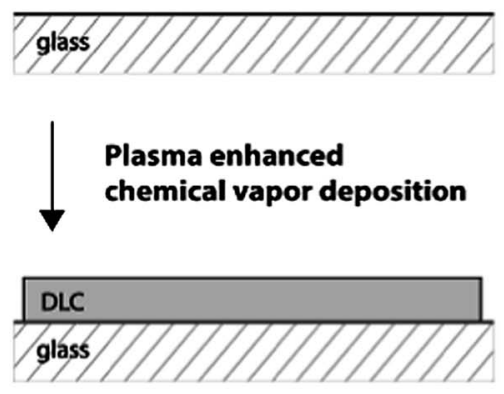

(c) Silanization (b) Writing process

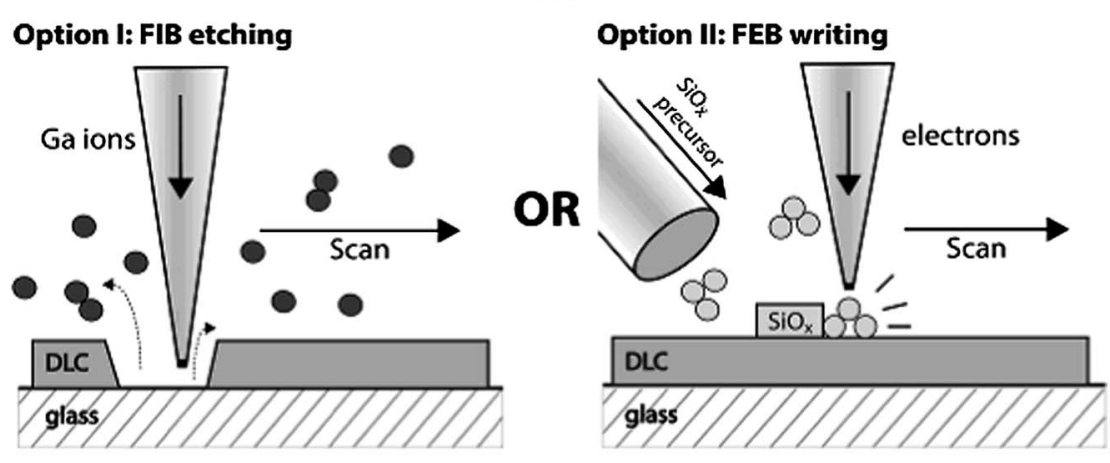

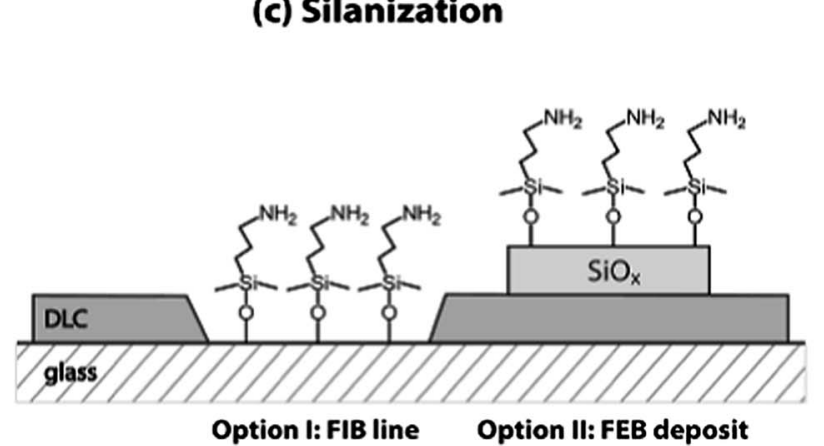

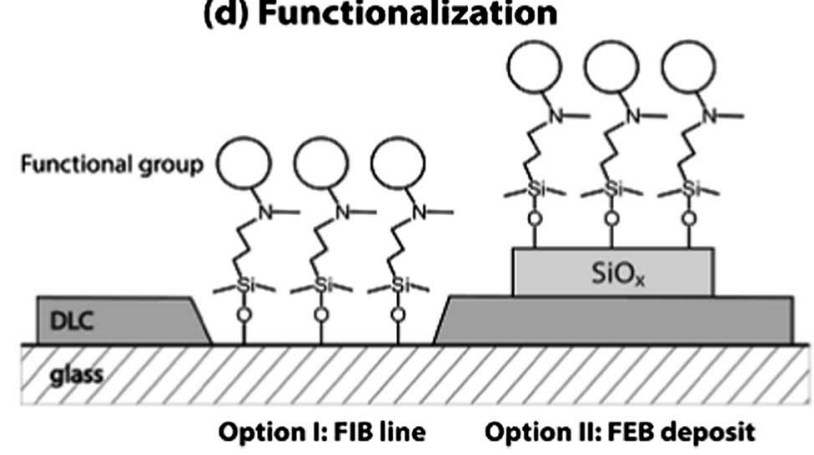

Fig. 1 Illustration of the fabrication process. (a) The glass surface is coated with DLC by plasma enhanced physical vapour deposition. (b) The glass is patterned by either focused electron beam-induced deposition or ion beam induced etching. (c) Surface activation by silanization. (d) Functional groups are covalently linked to the activated structures. 
Prior to the coating process, the glass slide substrates are cleaned by an Ar plasma for $15 \mathrm{~min}$ at a bias voltage of $-400 \mathrm{~V}$. Immediately after the plasma cleaning treatment, the DLC film is deposited by plasma activated chemical vapor deposition at a pulsed DC bias voltage of $-600 \mathrm{~V}$ for $11 \mathrm{~min}$ to reach a film thickness of $\sim 60 \mathrm{~nm}$. The ratio of the flow rates of argon and acetylene is set at $\mathrm{Ar}: \mathrm{C}_{2} \mathrm{H}_{2}=3: 2$ at a constant pressure of $3 \times$ $10^{-3}$ mbar. The purity of argon and acetylene gasses is 5.0 and technical grade, respectively.

Focused electron beam writing. The local deposition of siliconoxide $\left(\mathrm{SiO}_{x}\right)$ is done using focused electron beam-induced deposition (see Fig. 1b). The lithography platform is a focused ion beam (FIB)/scanning electron microscope, SEM (Lyra-XM FIB/SEM dual beam microscope, TESCAN, CZ) equipped with a Schottky field emission cathode in combination with a gallium FIB column. The precursor for the deposition of $\mathrm{SiO}_{x}$ is 2,4,6,8,10-pentamethylcyclopentasiloxane, a volatile liquid at room temperature. The vapour is introduced into the sample chamber close to the target through a multigas injection system. The electron source is operated between $10 \mathrm{keV}$ and $30 \mathrm{keV}$. The beam currents are measured with a Faraday cup and vary between $80 \mathrm{pA}$ and $2 \mathrm{nA}$. The minimum spot size is around $3.5 \mathrm{~nm}$. The sample and sample chamber are cleaned using a XEI Scientific Evactron 25 De-Contaminator for 10$15 \mathrm{~min}$. The background pressure is better than $5 \times 10^{-6} \mathrm{mbar}$, the chamber pressure during writing is around $4 \times 10^{-5}$ mbar. Patterns are defined using TESCANs proprietary software without the use of a beam blanker.

Focused ion beam etching. A Lyra-XM FIB/SEM dual beam microscope is used for focused $\mathrm{Ga}^{+}$ion beam patterning. Ion beam currents vary between $1 \mathrm{pA}$ for the smallest details and $10 \mathrm{nA}$ for larger details and the $\mathrm{Ga}^{+}$ion beam is operated at 30 $\mathrm{kV}$. The patterns are defined using TESCANs proprietary software. The smallest ion beam spot sizes are in the order of $50 \mathrm{~nm}$ (as specified by the manufacturer). The depth of the etched pattern is defined by varying the number of scans.

Surface activation by silanization. The selective functionalization is achieved by covalently linking an amine-containing silane, 3-aminopropyl-dimethyl-ethoxysilane, APDMES, (AcrosOrganics) to either the e-beam deposited $\mathrm{SiO}_{x}$ (option II, Fig. 1c) or the glass exposed by the ion beam etching (option I, Fig. 1c). Toluene $(60 \mathrm{ml})$ is heated to $65-70{ }^{\circ} \mathrm{C}$, APDMES is added at $\sim 1 \%$ $\mathrm{v} / \mathrm{v}$ and the solution is sonicated for $1 \mathrm{~min}$. The patterned glass cover slide is placed in the solution for $10 \mathrm{~min}$, after which it is rinsed using toluene and ethanol. The procedure provides an activated glass surface with free amino groups (Fig. 2, blue), which are ready for subsequent functionalization steps.

Labeling of the silane layer. The direct labeling of the patterned and silanized glass is shown schematically in Fig. 2a. The sample is immersed for $10 \mathrm{~min}$ at room temperature in a $100 \mathrm{nM}$ solution of ATTO655- $N$-hydroxysuccinimide, ATTO655NHS (ATTO-TEC GmbH) and $100 \mathrm{mM}$ aqueous $\mathrm{NaHCO}_{3}$ buffer (pH 8). For better solubility and chemical stability of the NHSester, $10 \mathrm{nmol}$ of the dye is pre-dissolved in water free dimethylsulfoxide and diluted in the $\mathrm{NaHCO}_{3}$ buffer. After the labeling the structure is rinsed with ethanol and MilliQ water.

Surface passivation with polyethylene glycol (PEG). Unspecific and noncovalent binding of functional molecules (e.g., fluorophores) can occur on the e-beam deposited $\mathrm{SiO}_{x}$ or on the glass exposed by the focused ion beam etching. To assure that the patterned areas are decorated only with specifically (covalently) bound molecules, the patterned areas are passivated. We use polyethylene glycol (PEG) as passivation layer, which is depicted schematically in Fig. 2b (dark blue). The silanized microscope cover slide is incubated at room temperature with a solution of $1 \mathrm{mg} \mathrm{ml}^{-1}$ biotin-PEG-succinimidyl valerate (MW 5000, Laysan Bio, Arab, AL) and $6 \mathrm{mg} \mathrm{ml}^{-1}$ methoxy-PEG-succinimidyl valerate (MW 5000, Laysan Bio, Arab, AL) dissolved in $100 \mathrm{mM} \mathrm{NaHCO}_{3}$ buffer at pH 8. After incubation overnight any unbound PEG/PEG-biotin is removed by rinsing with MilliQ water. The pegylated sample is blow dried and stored in a desiccator.

Surface passivation with BSA. The patterned glass can also be prepared for labeling using the unspecific binding of bovine serum albumin (BSA) on glass (Fig. 2, dark blue). For this functionalization and passivation strategy the unsilizanized (a) Labeling without passivation

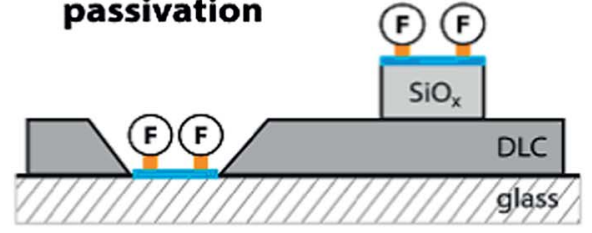

(b) Labeling with passivation

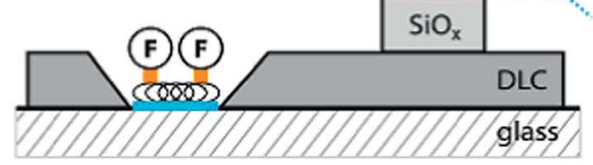

NHS-dye

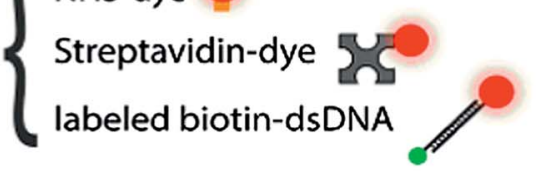

$\mathrm{NH}_{2}-\mathrm{NHS}$-Coupling

Biotin-Streptavidin-Interaction

BSA/BSA-Biotin

PEG-NHS/PEG-Biotin-NHS

Fig. 2 Schematic representation of the labeling strategies, without (a) and with (b) passivation. The dimensions are not to scale. 
structure is incubated at $4{ }^{\circ} \mathrm{C}$ with a $6.25 \mu \mathrm{g} \mathrm{ml}{ }^{-1} \mathrm{BSA}$ and $1.25 \mu \mathrm{g} \mathrm{ml}^{-1}$ BSA-biotin in PBS overnight. After passivation it is rinsed three times with PBS.

Labeling of the passivation layer. The patterned samples passivated with PEG/PEG-biotin or BSA/BSA-biotin are labeled with the following fluorophore derivatives: Cy5-streptavidin (Invitrogen) or pre-annealed dsDNA labeled with ATTO655 (Fig. 2b). Using direct streptavidin labelling, the passivated glass is exposed to $5 \mathrm{nM}$ solution of Cy5-streptavidin dissolved in PBS for $2.5 \mathrm{~min}$ at room temperature. In the case of labeling with dsDNA, samples are incubated with $0.2 \mathrm{mg} \mathrm{ml}^{-1}$ neutravidin (Invitrogen) for $10 \mathrm{~min}$. Unbound neutravidin molecules are removed by washing the structure with PBS buffer, before incubating it with $1 \mathrm{nM}$ dye-labeled DNA for $5 \mathrm{~min}$. After the labeling, all structures are washed 3 times in PBS and stored in PBS.

DNA annealing. To specifically label the patterned areas with DNA, we use two complementary 40-mer oligonucleotides. One contains the linking biotin-unit, referred to as DNA1 (biotin-5' CGT ATA GCT ATG CAA TAT AAG TGT AAG GAA TCG AAT ATT A3 as received from IBA, Germany). The other contains the fluorophore, referred to as DNA2 (ATTO655-C6-5'-TAA TAT TCG ATT CCT TAC ACT TAT ATT GCA TAG CTA TAC G-3'; as received from IBA, Germany). The single-stranded, lyophilized DNA is resuspended in MilliQ at a concentration of $100 \mu \mathrm{M}$ and preannealed in a heating block (Eppendorf, Mastercycler Pro). For this $100 \mu \mathrm{L}$ of $1 \mu \mathrm{M}$ solution of DNA1 and DNA2 is heated to $98{ }^{\circ} \mathrm{C}$ for $4 \mathrm{~min}$ and cooled to $4{ }^{\circ} \mathrm{C}$ at a rate of $1{ }^{\circ} \mathrm{C} \mathrm{min}{ }^{-1}$ in an annealing buffer (500 mM sodium chloride, $20 \mathrm{mM}$ TRIS-HCl, $1 \mathrm{mM}$ EDTA, $\mathrm{pH}=8$ ).

Imaging buffers. All measurements are performed at room temperature working in an aqueous imaging buffer, based on phosphate-buffered saline (PBS) at $\mathrm{pH}$ 7.4. Experiments with ATTO655 are carried out in PBS only. When the fluorophore Cy5 is used, $1 \mathrm{mM}$ Trolox is added and oxygen is removed from the buffer using an enzymatic oxygen scavenging system ${ }^{3,4}$ consisting of $0.1 \mathrm{mg} \mathrm{ml}^{-1}$ glucose oxidase, $40 \mu \mathrm{g} \mathrm{ml}^{-1}$ catalase, $0.2 \mathrm{mM}$ tris (2-carboxyethyl)phosphine hydrochloride (TCEP), $0.1 \mathrm{~g} \mathrm{ml}^{-1}$ glucose and $10 \%(\mathrm{v} / \mathrm{v})$ glycerine.

\subsection{Sample characterization}

Ensemble absorption and fluorescence spectroscopy. Absorption spectra in the visible and ultraviolet spectral range are recorded using a spectrophotometer (Jasco, V-640). The patterned cover slides are placed perpendicular to the beam path. Emission spectra are taken using a spectrofluorometer (Jasco, FP-8300) with an angle of 90 degree between the excitation and detection path. Both excitation and detection are done under an angle of 45 degrees with respect to the sample surface.

Atomic force microscopy. The topology of patterned samples is inspected using an atomic force microscope (Nanoscope IIIa, Digital Instruments), operated in tapping mode. An uncoated ndoped Si cantilever (Veeco Instruments SAS) with a spring constant of $20-80 \mathrm{~N} \mathrm{~m}^{-1}$ and a characteristic frequency of 300$366 \mathrm{kHz}$ was used.

Total internal reflection fluorescence imaging. Total internal reflection fluorescence (TIRF) imaging is carried out on an inverted microscope body (Olympus IX-71) equipped with an oil immersion objective lens (UPlanSApo $100 \times$, NA 1.4 Oil, Olympus). Additional lenses are inserted to achieve an overall magnification of $160 \mathrm{X}$. Under these conditions, one pixel images an area of $100 \times 100 \mathrm{~nm}^{2}$ on the sample. ATTO655 and Cy5 are excited with a $637 \mathrm{~nm}$ solid state laser (Obis, Coherent) at an intensity of $\sim 0.2 \mathrm{~kW} \mathrm{~cm}{ }^{-2}$. The laser beam is spectrally filtered using a cleanup filter (zet637/10×, Chroma) and coupled into the microscope objective via a dualband beamsplitter (z532/642rpc, Chroma). Fluorescent light passing through an emission filter (ET700/75m, Chroma) is detected using a CCD camera with an electron multiplier (ImagEM, Hamamatsu). TIRF or low-angle oblique (LAO) ${ }^{35}$ illumination is used to minimize the fluorescence background. For static
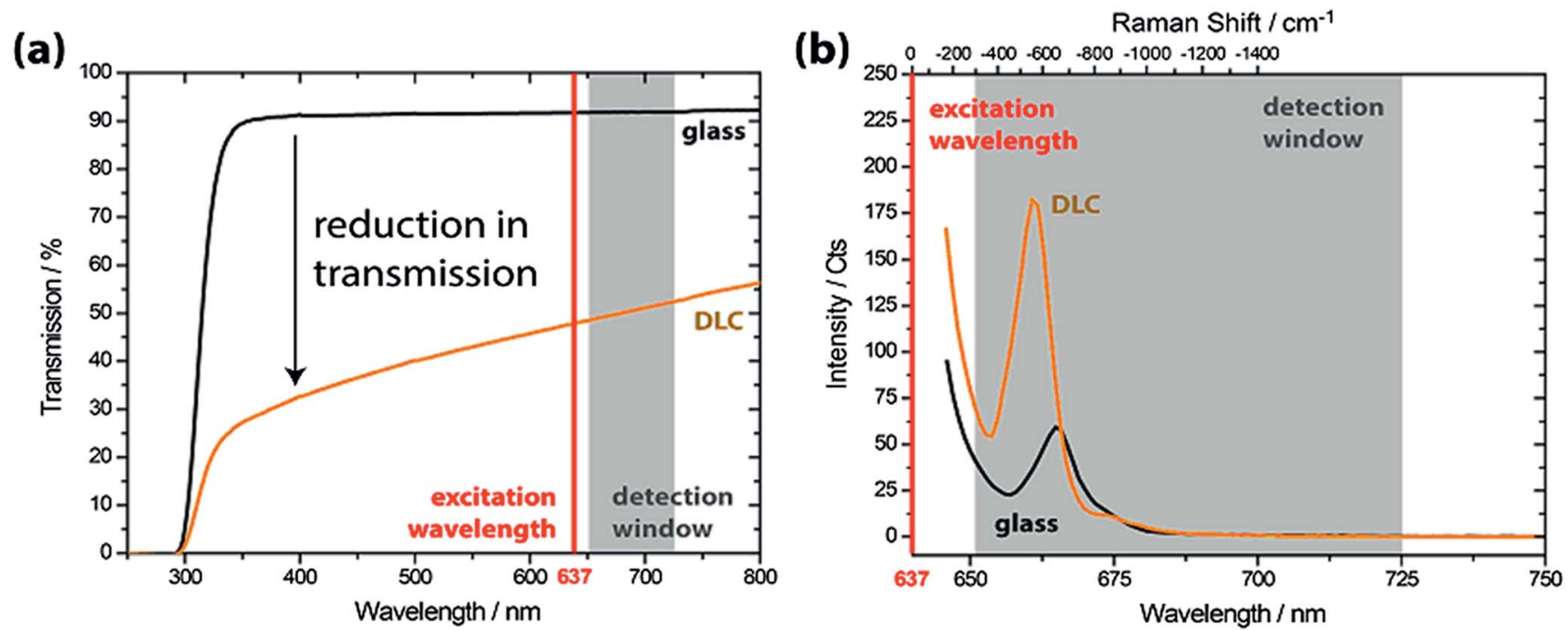

Fig. 3 Transmission (a) and emission (b) spectra of DLC coated glass. The detection window (grey) and excitation wavelength (red) being applied for the TIRF experiments are indicated in the graphs. 


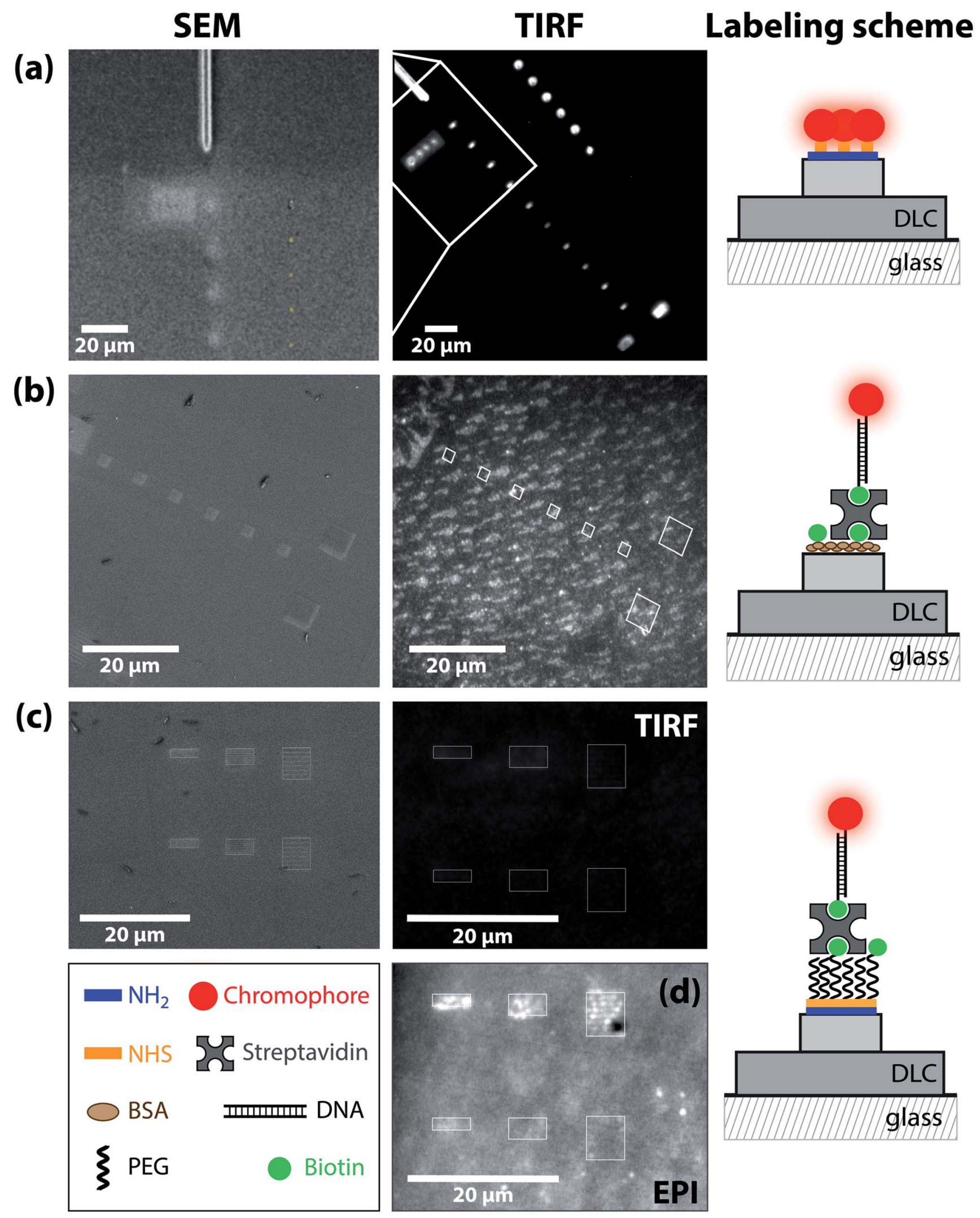

Fig. $4 \mathrm{FEB} \mathrm{SiO}_{x}$ structures characterized by SEM and TIRF imaging. (a) The activated surface is labeled directly with ATTO655-NHS. (b) The SiO ${ }_{x}$ is passivated with biotin/BSA and labeled with ATTO655-linked, biotinylated DNA. (c) The $\mathrm{SiO}_{x}$ is passivated with biotin/PEG via neutravidin and labeled with ATTO655-linked, biotinylated DNA. The pattern is only weakly visible in TIRF. (d) The same pattern from (c) is visible in epifluorescence. 
images, 100 frames are recorded at a frame rate of $10-30 \mathrm{~Hz}$ and averaged to suppress background noise.

Patterned cover slides are imaged with the patterned side facing away from the objective. A microscopic chamber gasket (LabTek, Nunc) is placed on top of the glass to enable the addition of the imaging buffer and the removal of oxygen.

\section{Results and discussion}

Before using DLC-coated glass for the assembly of fluorescent nanostructures, we characterized the optical properties. Fig. 3 shows the transmission and emission spectra of uncoated and DLC coated cover slides obtained with an optical spectrophotometer. As observed in Fig. 3a, the transmission is about $90 \%$ for the uncoated glass cover slide in the whole visible/nearinfrared range. In comparison, a DLC-coated cover slide has a transmission of about 50\% (at $637 \mathrm{~nm}$, the excitation wavelength in TIRF imaging). The emission properties of the DLC coated glass are studied by exciting the sample with $637 \mathrm{~nm}$ light in a spectrofluorometer. As reported in the literature, ${ }^{36}$ the coating itself shows no fluorescence, but a detectable signal contribution due to Raman scattering by the glass slide is seen, which is further enhanced and shifted by the DLC coating (Fig. 3b).

In addition to its optical activity, the DLC coating will act as a spacer in TIRF imaging. TIRF imaging relies on the evanescent, near-field wave that is generated at the glass interface. Since the evanescent field decays exponentially from the interface (in about 100-200 $\mathrm{nm}$ ), the DLC coating effectively reduces the excitation of fluorophores sitting on top of it. Based on these observations, we expect the imaging signal for patterns prepared with MACE-ID to be limited when combined with a DLC coating.

Fig. 4a shows the results of the functionalization using an adaption of the MACE-ID strategy, i.e. the labeling of electron beam written nanostructures on a coating (Fig. 1b, option II). $\mathrm{SiO}_{x}$ squares deposited on top of the diamond-like carbon (DLC) are faintly visible in the SEM image, as the contrast between the DLC and $\mathrm{SiO}_{x}$ thin film is low. The sample is silanized with 3aminopropyl-dimethyl-ethoxysilane (APDMES) and labeled with the fluorophore ATTO655-NHS. When imaged in TIRF mode, the contrast is high. The background intensities in the image are low, while the $\mathrm{SiO}_{x}$ deposits are clearly visible.

We observe in our experiments that, when patterned glass is silanized and functionalized directly, nonspecific binding occurs. On the locally exposed surface there are not only fluorophores covalently bound to the silanes (as intended), but also fluorophores that stay on the glass surface through physisorption or chemisorption. To prevent unspecific and noncovalent binding of fluorophores to the surface, we first passivated the $\mathrm{SiO}_{x}$ deposits with BSA (Fig. 4b). Fig. 4b shows a passivated sample with BSA/BSA-biotin and subsequent labeling with ATTO655-linked dsDNA oligomers, immobilized via neutravidin-biotin interactions. The SEM image shows the $\mathrm{SiO}_{x}$ squares written with the electron beam, but TIRF microscopy reveals a significant background signal and a poor signal from the labeled structures. In addition, fluorescent islands are visible outside the patterned area, suggesting that aggregates of the BSA/DNA-fluorophore are present on DLC.
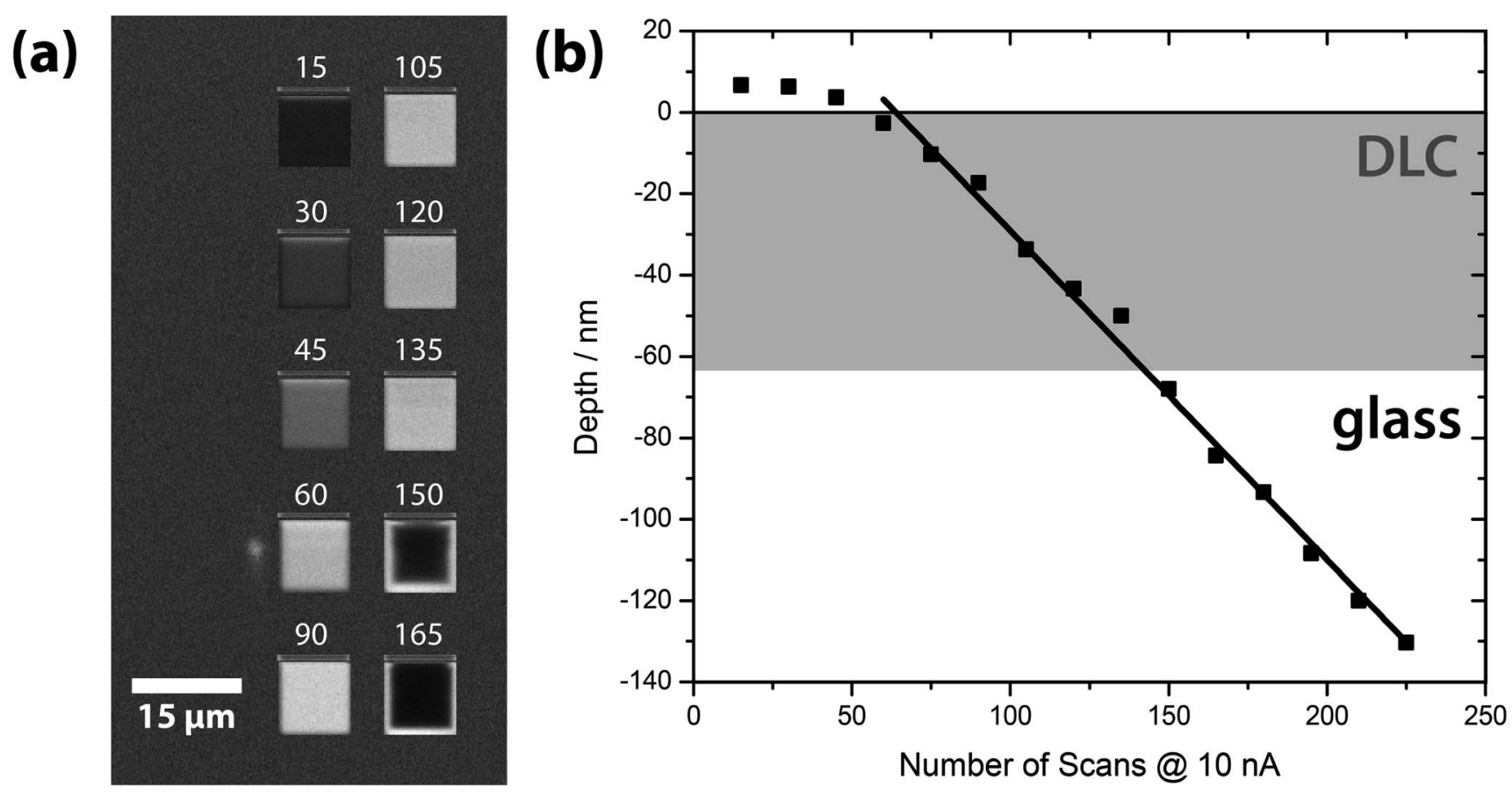

Fig. 5 The etched depth as function of the number of scans. (a) SEM image showing a series of 10 by $10 \mu$ m squares with increasing amount of scans. From the contrast reversal it is concluded that the DLC coating is fully removed at 150 scans. (b) Depth profile of the corresponding, etched terraces measured using the AFM. The DLC layer (depicted in grey) is about $60 \mathrm{~nm}$ thick. From about 50 scans onwards DLC is removed from the glass and shows a linear sputtering rate of about $-0.8+/-0.02 \mathrm{~nm} / \mathrm{scan}$. 
Fig. 4c shows the results of the second approach, in which we passivate the $\mathrm{SiO}_{x}$ deposits with PEG/PEG-biotin and label them with ATTO655-linked dsDNA via neutravidin-biotin interaction. The
SEM image shows two groups of deposited $\mathrm{SiO}_{x}$ lines written by MACE-ID with 4000 and 8000 scans each. The $50 \mathrm{~nm}$ wide lines are spaced at 800, 400 and $250 \mathrm{~nm}$. TIRF microscopy shows no (a)

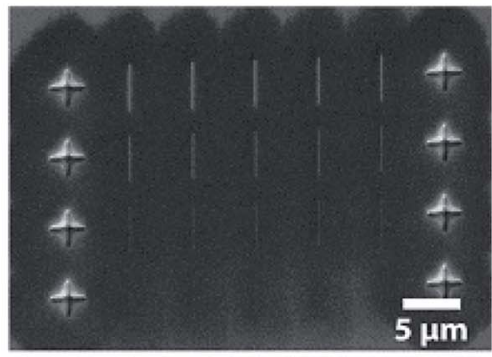

(b)

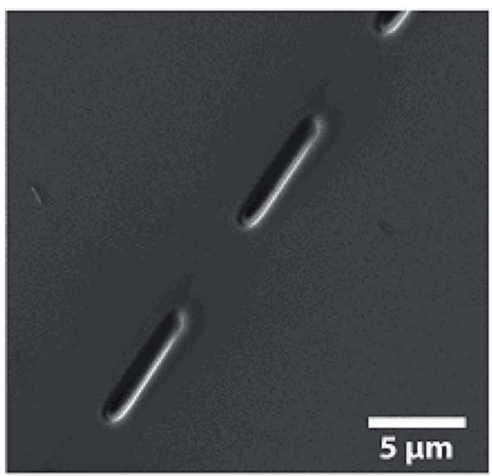

(c)

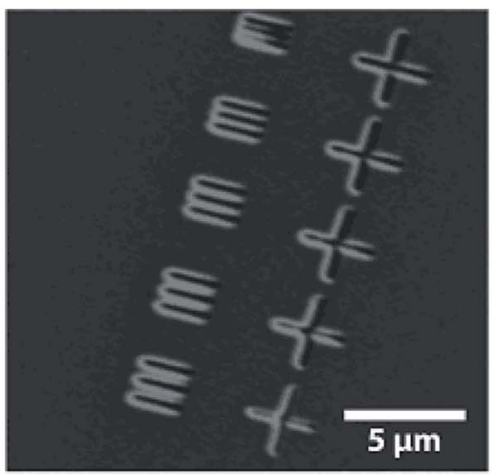

(d)

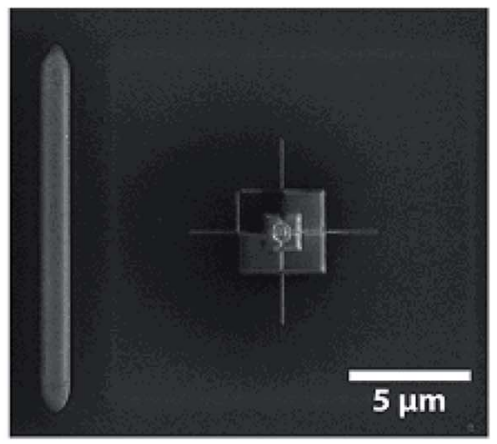

\section{TIRF}
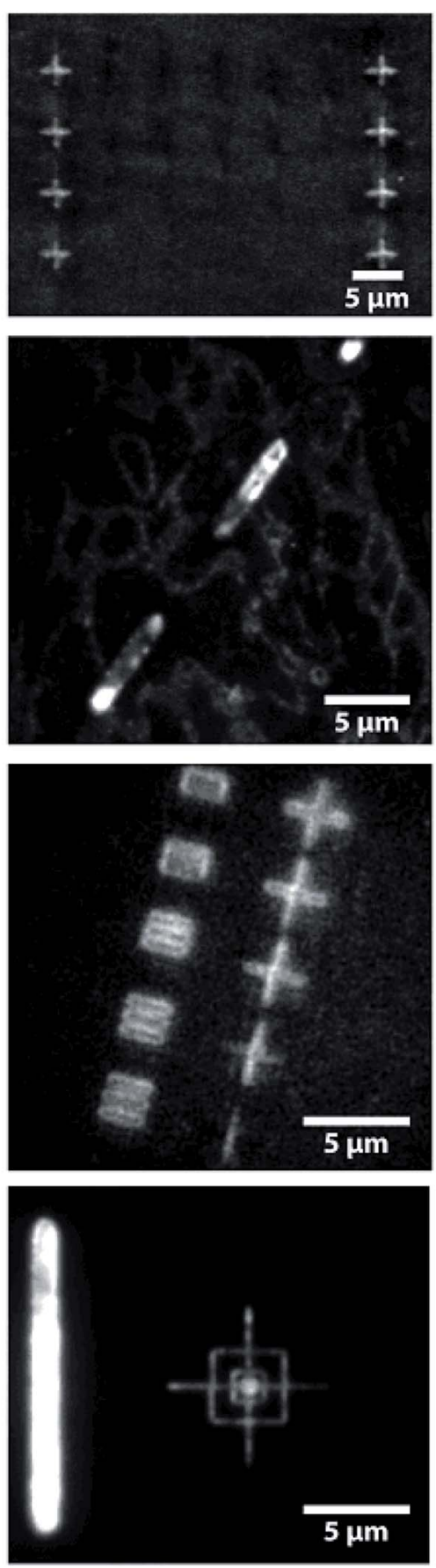

\section{Labeling scheme}
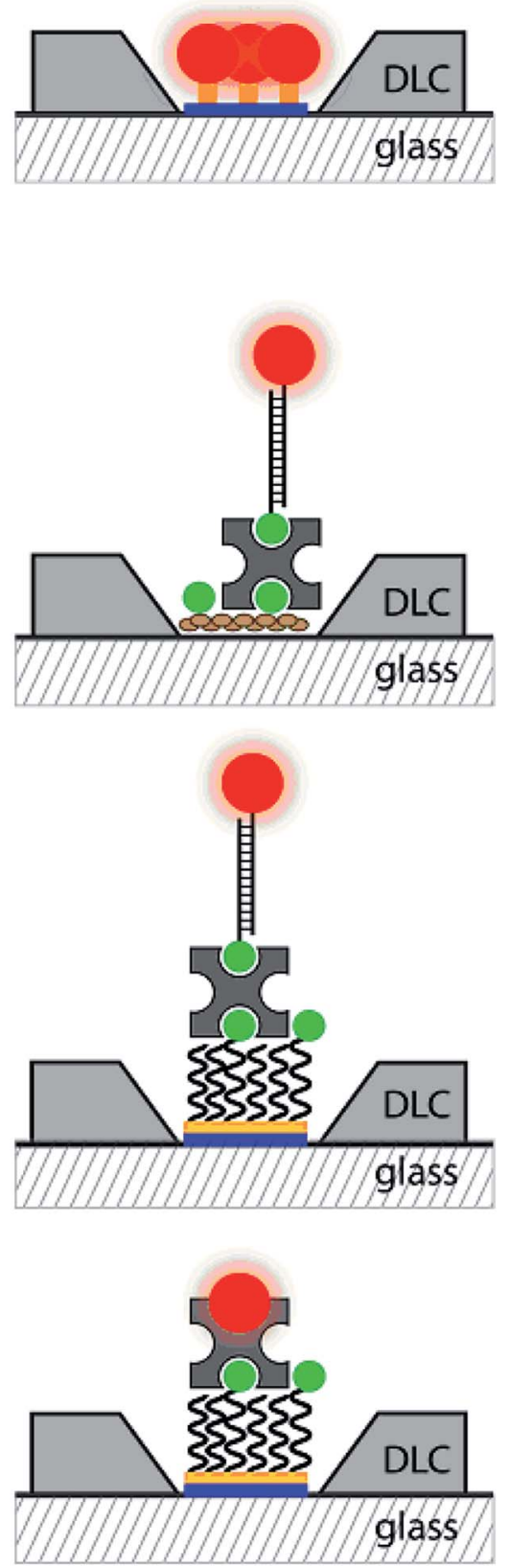

$\mathrm{NH}_{2}=\mathrm{NHS} \bigcirc \mathrm{BSA} \xi$ PEG $\bigcirc$ Biotin

Streptavidin

Chromophore

Inسm血 DNA

Fig. 6 Patterns etched with the focused ion beam, characterized using SEM and TIRF imaging. Activated surfaces were labeled (a) directly with ATTO655-NHS or $(b-d)$ with a passivation layer in between. Making use of biotin-neutravidin interactions, the patterns were labeled with (b) ATTO655-biotinylated DNA on the BSA- or (c) PEG-surface, respectively. (d) Cy5 is bound to streptavidin, with a ratio of $4: 3$ for PEG/PEG-biotin, respectively. 
fluorescent background around the written patterns, but also shows no detectable signal of the labeled deposits themselves. Toverify that the $\mathrm{SiO}_{x}$ are labeled, the written glass slide is inverted and inspected in epi-fluorescent mode. Although the signal-to-noise ratio is poor (due to the buffer layer used in epi-fluorescence mode), Fig. 4d indeed shows that the $\mathrm{SiO}_{x}$ patterns are labeled via biotin-neutravidin in combination with PEG as the passivation layer.
The results show that the BSA passivation layer physisorbs to the surface and does not selectively bind to the $\mathrm{SiO}_{x}$ deposits. And while the PEG/PEG-biotin is bound covalently to the activated surface and allows controlled and selective labeling, from Fig. 4c it is clear that the $\mathrm{SiO}_{x}$ deposits are not observed in TIRF. From these results we conclude that it is caused by the DLC coating and the PEG/PEG-biotin; together they place the

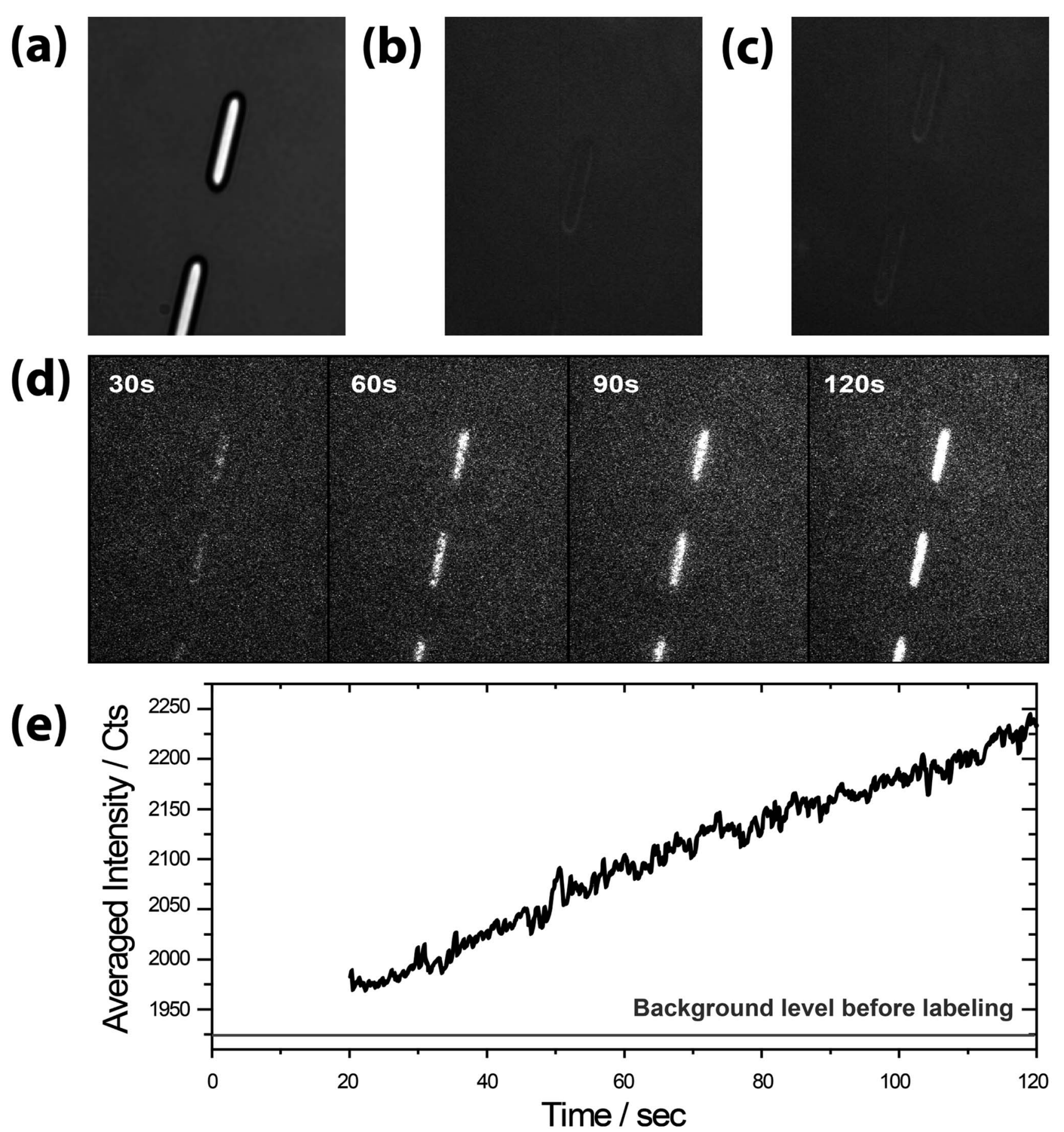

Fig. 7 Specific labeling by ATTO655-labeled DNA via biotin-neutravidin interaction on a FIB patterned glass coated with PEG/PEG-biotin. (a) Bright field image. TIRF image of the same surface exposed to (b) $2 \mathrm{nM}$ ATTO655-labeled DNA, (c) $0.2 \mathrm{mg} \mathrm{ml}^{-1} \mathrm{neutravidin}$ and (d) $2 \mathrm{nM}$ ATTO655-labeled DNA after incubation with neutravidin. Biotinylated DNA binds exclusively after addition of neutravidin to the surface. (e) The intensity averaged over $512 \times 512$ pixels as a function of time, showing the increasing labeling with incubation time. 
fluorophore relatively far from the glass interface and outside the evanescent field of excitation. This explains the absence of fluorescence intensity in Fig. 4c. In addition to this, the DLC coating attenuates the fluorescence signal of any labeled structure that sits on top of the coating due to its optical properties (as shown in Fig. 3).

To exploit the optical properties of the DLC coating (rather than to be limited by it) we locally remove the DLC using a focused ion beam (Fig. 1b, option I). If a pattern is defined in the DLC layer, the DLC layer itself helps to reduce the background signal of unspecifically bound fluorophores. Based on the data in Fig. 3a we conclude that the coating reduces the background signal significantly compared to the signal of actively etched patterns on glass. Fig. 5 shows the etched depth of 10 by $10 \mu \mathrm{m}^{2}$ squares as a function of the number of scans at a beam current of $10 \mathrm{nA}$. The thickness of the DLC coating is estimated from the SEM images; when the glass surface is exposed the contrast reverses due to charging. In Fig. 5a it is observed that this happens at 150 scans. A characterization using AFM reveals the depth profile within the structure as a function of the sputtering dosis (Fig. 5b). For a small amount of scans there is some deposition, rather than etching. We speculate that this is due to a local roughening of the surface, combined with deposition due to minor contamination in the vacuum system. However, etching is observed with proceeding scans (etch rate is about $-0.8+/-$ $0.02 \mathrm{~nm} / \mathrm{scan})$. This data shows that 150 scans are sufficient to fully etch through the $\sim 60 \mathrm{~nm}$ DLC layer and expose the glass.

Fig. 6 shows patterns etched into the DLC coating using the focused ion beam etching of the DLC coating, imaged using the SEM and (after functionalization) TIRF microscopy. We show functionalization without (Fig. 6a) and with glass passivation (Fig. 6b-d). The SEM images (Fig. 6a) reveal regularly spaced lines and crosses. Investigating the sample with TIRF microscopy after labeling with ATTO655, it is observed that only the crosses are bright, while the lines remain dark. This is because the lines are not etched through the coating and the underlying glass is not exposed. Comparing the intensities of the functionalized crosses and the DLC background, we observe high contrast and a low fluorescent background. In Fig. 6b, BSA/BSA-biotin is used to passivate the exposed glass surface and the lines are labeled with ATTO655-linked dsDNA oligomers, which are immobilized via neutravidin-biotin interactions. The SEM image shows a series of lines of $400 \mathrm{~nm}$ wide and around $7.5 \mu \mathrm{m}$ long. Although TIRF microscopy reveals that the pattern is labeled with fluorophores, other features are visible in the background as well. These features are most likely BSA-aggregates on the DLC coating, as have been observed also for the MACE-ID structures in Fig. $4 \mathrm{~b}$.

When the pattern is passivated and silanized with PEG/PEGbiotin (Fig. 6c and d), the passivation layer is specifically bound to the silanized patterns by $\mathrm{NH}_{2}-\mathrm{NHS}$-binding and unselective functionalization is not observed. When the PEG/PEG-biotin is labeled with DNA-ATTO655 (Fig. 6c) or streptavidin-Cy5 (Fig. 6d) the pattern is clearly visible. An improved signal-to-noise ratio can be achieved for streptavidin-Cy5, since it is easily excited on the TIRF microscope due to its height above the glass surface.

To verify that the biotin-streptavidin interaction is selective and specific, we expose a silanized PEG-surface to biotinylated
DNA. Fig. 7a shows a bright field image of DLC coated glass with FIB-etched lines. Fluorescence is not observed if there is only biotinylated ATTO655-DNA (Fig. 7b) or neutravidin (Fig. 7c) on the surface. Only after incubation with neutravidin binding of biotinylated DNA to the surface is observed, seen by the intensity increase over time (Fig. 7d-e). The underlying kinetics follows a simple binding as described for a biotin-avidin interaction. ${ }^{37}$ This demonstrates that the nanostructures are selectively labeled.

\section{Conclusion}

We present a novel approach to combine the bottom-up assembly and high-resolution lithography to position functional molecules on glass. The glass is coated with a $60 \mathrm{~nm}$ thick diamond-like carbon film. A pattern is defined by either writing $\mathrm{SiO}_{x}$ on the film with a focused electron beam, or exposing the underlying glass by focused ion beam etching. The patterns are silanized and labeled with a variety of functional groups, such as (but not limited to) ATTO655 and Cy5. The approach enables imaging of the patterns with TIRF microscopy. We demonstrate that the labeling is selective and that the patterns can be imaged with high signal-to-noise ratios.

The approach enables us to both position functional groups on a surface and to verify their positions and surface concentrations accurately. Our approach promises to benefit, amongst others, single-molecule biophysics studies and studies using zero-mode waveguides. ${ }^{38,39}$ The specific positioning of single fluorescently labeled molecules, such as DNA, proteins, or viruses simplifies data analysis and provides a solution for the problem of unspecific binding in single-molecule experiments.

\section{Acknowledgements}

E. P. acknowledges a DFG fellowship (PL696/2-1). B.L.F. was supported by the Ministry for Science and Education (Gravity program). W.F.v.D. was supported by a Veni grant (Grant no. 10684 - STW-Utrecht) and the Zernike Institute for Advanced Materials. T. C. was supported by the Zernike Institute for Advanced Materials and the Centre for Synthetic Biology (University of Groningen). We are grateful to A. M. van Oijen and B. Poolman for support and access to laboratory facilities. We finally thank M. Punter and V. Krasnikov for help with data analysis and experiments.

\section{References}

1 A. Chan, R. P. Orme, R. A. Fricker and P. Roach, Adv. Drug Delivery Rev., 2013, 65, 497-514.

2 H. E. Huxley, Science, 1969, 164, 1356-1366.

3 Y. Salomon, M. Lin, C. Londos, M. Rendell and M. Rodbell, J. Biol. Chem., 1975, 250, 4239-4245.

4 M. Lin, Y. Salomon, M. Rendell and M. Rodbell, J. Biol. Chem., 1975, 250, 4246-4252.

5 M. Rendell, Y. Salomon, M. Lin, M. Rodbell and M. Berman, J. Biol. Chem., 1975, 250, 4253-4260.

6 D. A. Leigh, J. K. Wong, F. o. Dehez and F. Zerbetto, Nature, 2003, 424, 174-179. 
7 R. Eelkema, M. M. Pollard, J. Vicario, N. Katsonis, B. S. Ramon, C. W. Bastiaansen, D. J. Broer and B. L. Feringa, Nature, 2006, 440, 163-163.

8 B. Lewandowski, G. De Bo, J. W. Ward, M. Papmeyer, S. Kuschel, M. J. Aldegunde, P. M. Gramlich, D. Heckmann, S. M. Goldup and D. M. D'Souza, Science, 2013, 339, 189-193.

9 S. J. van der Molen, J. Liao, T. Kudernac, J. S. Agustsson, L. Bernard, M. Calame, B. J. van Wees, B. L. Feringa and C. Schönenberger, Nano Lett., 2008, 9, 76-80.

10 C. Raimondo, N. Crivillers, F. Reinders, F. Sander, M. Mayor and P. Samori, Proc. Natl. Acad. Sci. U. S. A., 2012, 109, 1237512380.

11 B. K. Pathem, S. A. Claridge, Y. B. Zheng and P. S. Weiss, Annu. Rev. Phys. Chem., 2013, 64, 605-630.

12 G. London, K.-Y. Chen, G. T. Carroll and B. L. Feringa, Chem. - Eur. J., 2013, 19, 10690-10697.

13 D. Janssen, R. De Palma, S. Verlaak, P. Heremans and W. Dehaen, Thin Solid Films, 2006, 515, 1433-1438.

14 T. Kudernac, N. Katsonis, W. R. Browne and B. L. Feringa, J. Mater. Chem., 2009, 19, 7168-7177.

15 C. J. Barrett, J.-i. Mamiya, K. G. Yager and T. Ikeda, Soft Matter, 2007, 3, 1249-1261.

16 V. Balzani, A. Credi and M. Venturi, ChemPhysChem, 2008, 9, 202-220.

17 A. G. Curto, G. Volpe, T. H. Taminiau, M. P. Kreuzer, R. Quidant and N. F. van Hulst, Science, 2010, 329, 930-933.

18 O. Arcizet, V. Jacques, A. Siria, P. Poncharal, P. Vincent and S. Seidelin, Nat. Phys., 2011, 7, 879-883.

19 V. Auzelyte, C. Dais, P. Farquet, D. Grützmacher, L. J. Heyderman, F. Luo, S. Olliges, C. Padeste, P. K. Sahoo and T. Thomson, J. Micro/Nanolithogr., MEMS, MOEMS, 2009, 8, 021204-021210.

20 V. R. Manfrinato, L. Zhang, D. Su, H. Duan, R. G. Hobbs, E. A. Stach and K. K. Berggren, Nano Lett., 2013, 13, 15551558.

21 W. F. Van Dorp, X. Zhang, B. L. Feringa, T. W. Hansen, J. B. Wagner and J. T. M. De Hosson, ACS Nano, 2012, 6, 10076-10081.
22 C. M. Kolodziej and H. D. Maynard, Chem. Mater., 2012, 24, 774-780.

23 T. Baumgärtel, C. von Borczyskowski, H. Graaf and Beilstein, J. Nanotechnol., 2013, 4, 218-226.

24 L. Demers, D. Ginger, S.-J. Park, Z. Li, S.-W. Chung and C. Mirkin, Science, 2002, 296, 1836-1838.

25 K. Salaita, Y. Wang, J. Fragala, R. A. Vega, C. Liu and C. A. Mirkin, Angew. Chem., 2006, 118, 7378-7381.

26 S. K. Kufer, E. M. Puchner, H. Gumpp, T. Liedl and H. E. Gaub, Science, 2008, 319, 594-596.

27 W. Slingenbergh, S. K. de Boer, T. Cordes, W. R. Browne, B. L. Feringa, J. P. Hoogenboom, J. T. M. De Hosson and W. F. van Dorp, ACS Nano, 2012, 6, 9214-9220.

28 T. Ha and P. Tinnefeld, Annu. Rev. Phys. Chem., 2012, 63, 595-617.

29 E. Betzig, G. H. Patterson, R. Sougrat, O. W. Lindwasser, S. Olenych, J. S. Bonifacino, M. W. Davidson, J. LippincottSchwartz and H. F. Hess, Science, 2006, 313, 1642-1645.

30 S. T. Hess, T. P. Girirajan and M. D. Mason, Biophys. J., 2006, 91, 4258-4272.

31 M. J. Rust, M. Bates and X. Zhuang, Nat. Methods, 2006, 3, 793-795.

32 T. Cordes, M. Strackharn, S. W. Stahl, W. Summerer, C. Steinhauer, C. Forthmann, E. M. Puchner, J. Vogelsang, H. E. Gaub and P. Tinnefeld, Nano Lett., 2010, 10, 645-651.

33 J. Vogelsang, T. Cordes, C. Forthmann, C. Steinhauer and P. Tinnefeld, Proc. Natl. Acad. Sci. U. S. A., 2009, 106, 81078112.

34 R. Roy, S. Hohng and T. Ha, Nat. Methods, 2008, 5, 507-516. 35 Y. Sako, Imaging single molecules in living cells for systems biology, Mol. Syst. Biol., 2006, 2, DOI: 10.1038/msb4100100.

36 T. Sameshima and N. Andoh, Jpn. J. Appl. Phys., 2005, 44, 7305-7308.

37 J. R. Wayment and J. M. Harris, Anal. Chem., 2009, 81, 336342.

38 P. Hinterdorfer and A. Van Oijen, Handbook of singlemolecule biophysics, Springer, 2009.

39 P. Zhu and H. G. Craighead, Annu. Rev. Biophys., 2012, 41, 269-293. 